\title{
Extended Geometric Scaling from Generalized Traveling Waves
}

\author{
R. Peschanski* \\ Institut de Physique Théorique (IPhT), CEA/Saclay, 91191 Gif-sur-Yvette cedex, France ${ }^{\dagger}$
}

\begin{abstract}
We define a mapping of the QCD Balitsky-Kovchegov equation in the diffusive approximation with noise and a generalized coupling allowing a common treatment of the fixed and running QCD couplings. It corresponds to the extension of the stochastic Fisher and Kolmogorov-PetrovskyPiscounov equation to the radial wave propagation in a medium with negative-gradient absorption responsible for anomalous diffusion, non-integer dimension and damped noise fluctuations. We obtain its analytic traveling wave solutions with a new scaling curve and for running coupling a new scaling variable allowing to extend the range and validity of the geometric-scaling QCD prediction beyond the previously known domain.
\end{abstract}

\section{INTRODUCTION}

Geometric scaling is originally [1] an empirical scaling law satisfied by the data on deep-inelastic totally inclusive cross-sections. It may be translated as a property of the scattering amplitude

$$
T(L, Y)=T[\tau(L, Y)]
$$

where the scaling variable $\tau(L, Y)=L-v Y$ in the original formulation. The usual kinematic variables are $Y$, the total rapidity and $L=\log \left(Q^{2} / \Lambda^{2}\right)$ with $Q$ the photon virtuality and $\Lambda$ the Quantum Chromodynamics (QCD) reference scale at one-loop level.

On the theoretical ground, there exists known encouraging features of QCD in the saturation regime leading to approximate geometric scaling, but the situation is not yet settled. The good news were, for instance, that in the large- $N_{c}$ limit and in the mean-field approximation, we are led to consider the Balitsky-Kovchegov (BK) equation [2] which possesses the nice property [3] to be mapped, in the diffusive approximation, onto the Fisher-KolmogorovPetrovsky-Piscounov (F-KPP) equation $[4,5]$. This equation which has been widely studied in statistical physics is known to admit traveling waves as asymptotic solutions which translate into geometric scaling in QCD [1]. In fact one may enlarge that result to a whole class of equations, including the one considering the full QCD kernel [6] beyond the restriction to the first two derivatives. One may introduce the notion of "universality class" since the asymptotic properties of the solutions, including the approximate geometric scaling, do not depend either on initial conditions or on a precise form of the nonlinear part of the equation.

However, many questions remain unsolved. On a theoretical ground, there are quite a few problems to ensure analytical scaling properties, even in the simpler case of BK equation with running coupling, see e.g. the discussion of Ref.[7]. Even more puzzling is the discovery that introducing a small cut-off [8] to the FKPP or BK equation or adding a small white noise [9], i.e. for a stochastic (s)FKPP or (s)BK equation, which after all reflects a physical constraint relying on the finiteness and randomness of the number of gluons, predicts a strong violation of geometric scaling not seen on data. On the other hand, the same stochasticity applied to the running coupling case seems from indirect evidence either to preserve geometric scaling [10] or to delay its violation to much higher energy [11].

So, apart from the phenomenological problems, which we will discuss in a further publication, the aim of the present paper is on the general theoretical issue of geometric scaling from the BK nonlinear evolution equation, in particular for the running coupling case. Translating the problem in more mathematical terms, it seems that the "universality class" of the BK equation with runnning coupling has not yet been found or at least clarified. It is our goal to propose a solution to this problem, and explore its consequences which happen to give rise to an extended scaling property, as will become clear soon. In fact we will define the initial mathematical problem as the diffusive approximation of the (s)BK equation with generalized running coupling, extrapolating from the running or non-running case.

One starts by considering the following one-parameter family

$$
L^{n} \partial_{Y} N(L, Y)=\left\{A_{2} \partial_{L}^{2}+A_{1} \partial_{L}+A_{0}\right\} N(L, Y)-N^{2}(L, Y)+\sqrt{\kappa N(L, Y)} \nu(L, Y),
$$

where the case $n=1$ (resp. $n=0$ ) corresponds to the logarithmically running (resp. non-running) coupling in the (s)BK equation within the diffusive approximation $\left(1 / L^{n}\right.$ has been divided on both sides of $\left.(2)\right)$. Universality properties,

† URA 2306, unité de recherche associée au CNRS. 
independent from initial conditions and the form of the nonlinear damping, will provide the suitable identification of the universality class. In equation (2)

$$
\chi\left(-\partial_{L}\right) \sim A_{2} \partial_{L}^{2}+A_{1} \partial_{L}+A_{0}
$$

represents for our purposes the expansion up to two derivatives of the linear kernel, either for the FKPP equation (where $\chi(\gamma)=\gamma^{2}+1$ ) or for the diffusive approximation of the Balitsky, Fadin, Kuraev and Lipatov (BFKL) kernel [6] relevant for the BK equation. The strength of the noise term is specified by the phenomenological fudge factor $\kappa$, and $\nu(L, Y)$ is the standard white noise satisfying $\langle\nu\rangle=0$ and $\left\langle\nu(L, Y) \nu\left(L^{\prime}, Y^{\prime}\right)\right\rangle=\delta\left(Y-Y^{\prime}\right) \delta\left(L-L^{\prime}\right)$.

Investigating the properties of the general nonlinear evolution equation (2), the paper is organized as follows. In section 2, we show that Eq.(2) can be exactly mapped onto the extension of the stochastic Fisher and KolmogorovPetrovsky-Piscounov equation for the radial wave propagation in a medium with absorptive negative gradient, noninteger dimension and damped noise fluctuations when $n>0$. In section 3 , we derive new scaling solutions for arbitrary $n$ verifying the general recipe (1), defining for every value of $n$ a scaling variable $\tau(L, Y)$ and a new scaling curve $T(\tau(L, Y)$ ) valid in the forward front of the traveling wave in a region (in $L$ ) beyond but neighbouring the previously considered $[3,8]$ domain. By compatibility with those previous universal results, in section 4 we establish the universality forms, indexed by $n$, of the traveling wave solutions and focus on the physically interesting cases $n=0,1$. We check recovering known results for $n=0,1$ and find a geometric scaling parameterization extended in a larger kinematic domain and for the running case a new scaling variable. Summary and outlook can be found in section 5 .

\section{MAPPING}

The initial idea of our approach is to introduce a change of variables aiming at restoring the "standard" constant diffusion term, which determines the mathematical order of the (linear part of the) partial differential equation (2). In some sense one is guided by restoring the classical diffusion equation (or also "heat equation") $\partial_{T}=\partial_{X}^{2}+\cdots$, where $T$ is "time" and $X$ is "space". One then writes the Ansatz

$$
L=X^{\beta} ; \quad \partial_{L}=\frac{1}{\beta} X^{1-\beta} \partial_{X},
$$

with $\beta$ to be determined for our purpose.

After a straightforward transformation, Eq.(2) takes the form

$$
\begin{array}{r}
X^{n \beta} \frac{\partial N(X, Y)}{\partial Y}=\left\{\frac{A_{2}}{\beta^{2}} X^{2-2 \beta} \partial_{X}^{2}+\left(A_{2} \frac{1-\beta}{\beta^{2}} X^{1-2 \beta}+\frac{A_{1}}{\beta} X^{1-\beta}\right) \partial_{X}+A_{0}\right\} N(X, Y)- \\
-N^{2}(X, Y)+\sqrt{\frac{\kappa N(L, Y)}{\beta X^{\beta-1}}} \nu(X, Y)
\end{array}
$$

where the noise term has been conveniently renormalized such that $\left\langle\nu(X, Y) \nu\left(X^{\prime}, Y^{\prime}\right)\right\rangle=\delta\left(Y-Y^{\prime}\right) \delta\left(X-X^{\prime}\right)$.

Now, asking for a constant diffusion coefficient can easily be achieved by equalizing the powers of the first two terms of (5) namely

$$
n \beta=2-2 \beta \rightarrow \beta=\frac{2}{n+2}
$$

leading to the equation

$$
\begin{aligned}
& \frac{\partial N(X, Y)}{\partial Y}=\frac{A_{2}}{\beta^{2}} \partial_{X}^{2} N(X, Y)+\left(\frac{A_{2}(1-\beta)}{\beta^{2}} X^{-1}+\frac{A_{1}}{\beta} X^{-(1-\beta)}\right) \partial_{X} N(X, Y) \\
& +X^{-2(1-\beta)}\left(A_{0} N(X, Y)-N^{2}(X, Y)\right)+\sqrt{\frac{\kappa X^{-3(1-\beta)}}{\beta} N(X, Y)} \nu(X, Y)
\end{aligned}
$$

Introducing new variables

$$
T=\frac{A_{2}}{\beta^{2}} Y, \quad \text { and } U(X, T)=\frac{N}{A_{0}},
$$


Eq. (7) gets mapped onto the "sFKPP- type" equation

$$
\frac{\partial U(X, T)}{\partial T}=\partial_{X}^{2} U(X, T)+\left((1-\beta) X^{-1}+\gamma X^{-(1-\beta)}\right) \partial_{X} U(X, T)+\lambda X^{-2(1-\beta)}\left(U-U^{2}\right)+\sqrt{\mu X^{-3(1-\beta)} U} \nu(X, T)
$$

with

$$
\begin{aligned}
& \gamma=\beta \frac{A_{1}}{A_{2}}, \quad \lambda=\beta^{2} \frac{A_{0}}{A_{2}}, \quad \mu=\frac{\kappa}{\beta}, \\
& \left\langle\nu(X, T) \nu\left(X^{\prime}, T^{\prime}\right)\right\rangle=\delta\left(X-X^{\prime}\right) \delta\left(T-T^{\prime}\right) .
\end{aligned}
$$

Note that, as well-known, one may introduce an extra factor $\sqrt{1-U}$ in the noise term to ensure an absorptive boundary also at $U=1$. However, the effects of the noise are really important only in the dilute tail of the wave, while this modification allows to recover exactly the $1 \mathrm{~d}$ sFKPP equation for $\beta=1$.

A suggestive interpretation of Eq.(7) comes from the redefinitions (8). While the diffusion constant is by definition rescaled to 1 , the successive coefficient functions acquire the following meaning:

- $(1-\beta) X^{-1} \partial X$ is the radial Laplacian term corresponding to a medium of noninteger dimension $2-\beta$.

- $\gamma X^{-(1-\beta)}$ is, at $\beta=1$, the "drift" term which is now decreasing at large $X$ for $\beta \neq 1$ and thus no more absorbed by a redifinition of the "space" variable (see [3]). It may be interpreted as a kind of space-dependent rotation of the space-time frame.

- $\lambda X^{-2(1-\beta)}$ defines a dynamical birth-death coupling strength characterizing a medium equipped with a negative gradient; it has thus the important effect of a space-dependent "absorption" on the wave propagation.

- $\mu X^{-3(1-\beta)}$ is the new dynamical noise strength, strongly decreasing when $X$ gets large.

A qualitative interpretation of the hierarchical behaviour of these coefficients as a function of $X$ suggests a similar hierarchy of the expected effects on the solutions. Indeed, we will see that it leads to a $\beta$-dependent anomalous effective diffusion. Moreover, the fluctuation strength (asssociated to the quadratic correlators of $\nu(X, T)$ ) leads to an expected damping of the fluctuations and thus of the stochasticity effects. In the rest of the paper we will thus neglect the stochasticity, postponing its precise study to further work.

It is mathematically interesting to note that Eq.(7) possesses a well-defined limit $n \rightarrow \infty$, i.e. $\beta \rightarrow 0$, giving rise to the equation

$$
\partial_{T} U(X, T)=\partial_{X}^{2} U(X, T)+X^{-1} \partial_{X} U(X, T)+\lambda X^{-2} U(1-U)+\sqrt{\kappa X^{-3} U} \nu(X, T),
$$

which appears to be the radial sFKPP equation in a 2-dimensional medium characterized by a negative gradient corresponding to the birth-death factor $\lambda X^{-2}$. The noise term is also depressed by the factor $\kappa X^{-3}$ which defines the coupling strength of the noise correlator.

Hence, in general, for nonzero $\beta$ the constant (by construction) diffusive coefficient is larger than the effective birth rate, which is itself larger than the effect of the noise correlators. This hierarchy can be interpreted as due to a "radial" sFKPP equation in a medium of dimensionality between one and two with negative gradient and decreased noise. Propagation of waves in such a "medium" should and will affect the properties of the traveling solutions which we shall now examine.

\section{SCALING SOLUTIONS}

Inspired by the fundamental traveling-wave property of the standard FKPP equation, our strategy will be to focus on some region of the forward front and thus look for a scaling variable $\tau(L, Y)$ expressed in the new parameterization as $\bar{X}(X, T)$. It will lead to a solution of the form of a traveling wave $U(\bar{X})$, based on an ansatz valid in a range of "space" and "time" which will be made precise later on. Let us recall that in all the following we shall concentrate our study on the deterministic equation without noise. 


\section{Scaling Solution: First Order Approximation}

Let us initially introduce an Ansatz

$$
U=A e^{-a\left(X-v T X^{-\alpha}\right)^{b}}
$$

where the constants $a, b, \alpha$ and the "speed" $v$ have to be determined. Our strategy is to first solving Eq.(7) at leading order when $X \sim \bar{X}$ is large w.r.t. $v T X^{-\alpha}$ and in a second step going beyond this approximation. Using the convenient formulae

$$
\partial_{T} U=\partial_{T} \bar{X} \partial_{\bar{X}} U \quad \partial_{X} U=\partial_{X} \bar{X} \partial_{\bar{X}} U \quad \partial_{X^{2}} U=\partial_{X^{2}} \bar{X} \partial_{\bar{X}} U+\left(\partial_{X} \bar{X}\right)^{2} \partial_{\bar{X}}^{2} U
$$

we can write, within the same approximation $X \sim \bar{X}$

$$
\begin{aligned}
\frac{1}{U} \partial_{T} U & =\left(a b \bar{X}^{2 b-1}\right) v \bar{X}^{-\alpha} \\
\frac{1}{U} \partial_{X} U & =\left(-a b \bar{X}^{2 b-1}\right)\left(1+v \alpha T \bar{X}^{\alpha-1}\right) \\
\frac{1}{U} \partial_{X}^{2} U & =\left(a^{2} b^{2} \bar{X}^{4 b-2}+a b(1-2 b) \bar{X}^{3 b-2}\right)\left(1+v \alpha T \bar{X}^{\alpha-1}\right)^{2}+ \\
& +\left\{a b \bar{X}^{2 b-1}\right\}(1-2 b)(2-2 b) v T \bar{X}^{2 b-3}
\end{aligned}
$$

In a first step we solve the linear part of Eq.(7). As well-known [5], the forward front can be studied by restricting first to the solution of the linear equation since the nonlinear part has no direct effect (but important indirect selection effect) on the solution in this range. Selecting now leading orders when $X \sim \bar{X}$ is large w.r.t. $v T X^{-\alpha}$ we find an iterative solution of (7) by ordering in decreasing powers of $X$. Within the approximation $X \sim \bar{X}$ and inserting the relations (12) in (7) one realizes that the leading order should be obtained with terms proportional to $\bar{X}^{2 \beta-2}$.

Collecting the leading terms from all contributions one gets

$$
\begin{aligned}
\frac{1}{U} \partial_{T} U & : \operatorname{vab} \bar{X}^{2 b-\alpha-1} \\
\frac{1-\beta}{U X} \partial_{X} U & :-a b(1-\beta) \bar{X}^{2 b-2} \\
\gamma \bar{X}^{\beta-1} \frac{1}{U} \partial_{X} U & :-\gamma a b \bar{X}^{\beta+2 b-2} \\
\frac{1}{U} \partial_{X}^{2} U & : a^{2} b^{2} \bar{X}^{4 b-2}+a b(1-2 b) \bar{X}^{3 b-2} \\
\frac{1}{U} \lambda U & : \lambda \bar{X}^{2 \beta-2} .
\end{aligned}
$$

One thus gets a matching of the dominant contribution in $\bar{X}^{2 \beta-2}$ by the following relations

$$
\begin{array}{r}
\alpha=1-\beta ; \quad b=\frac{\beta}{2} \\
v a b=a^{2} b^{2}-\gamma a b+\lambda
\end{array}
$$

and thus finds two solutions for the parameter $a$, namely

$$
a_{ \pm}=\frac{1}{\beta}\left(v+\gamma \pm \sqrt{(v+\gamma)^{2}-4 \lambda}\right)
$$

Hence, as expected from solutions of a second-order equation, the general solution can be (except for the case $a_{+}=a_{-}$ see further on) expressed as

$$
U(X, T)=A_{+} e^{-a_{-}\left(X-v T X^{\beta-1}\right)^{\frac{\beta}{2}}}-A_{-} e^{-a_{+}\left(X-v T X^{\beta-1}\right)^{\frac{\beta}{2}}} .
$$

Looking to the solutions (15) one sees that one has the condition $v \geq 2 \sqrt{\lambda}$ in order to have real solutions, and thus the dominant speed is minimal when the condition

$$
v_{c}+\gamma=2 \sqrt{\lambda}=a_{c} \beta
$$

is satisfied, where $v_{c}$ (resp. $a_{c}$ ) are the critical speed (resp. critical slope). We recognize among the conditions (17) the same critical speed as the one determined by the standard method [3, 8] when $n=0,1$. Note however that the critical slope $a_{c}$ is $\beta$-dependent contrary to what is found with the Brunet-Derrida type of Ansatz [3, 8]. 


\section{Scaling Solution: Second Order Approximation}

The critical conditions are realized as a special solution for the "degenerate case" $a_{+}=a_{-}$corresponding to the Ansatz (where we do not add the possible second pure exponential term, as will be justified in the following section)

$$
U=A e^{-a_{c}\left(X-v_{c} T X^{\beta-1}\right)^{\frac{\beta}{2}}}\left(X-v_{c} T X^{\beta-1}\right)^{\frac{\beta}{2}},
$$

corresponding to a geometric scaling variable (restoring for a while the $L$ and $n$ dependence),

$$
\bar{X}=X-v_{c} T X^{\beta-1}=X-v_{c} T X^{-\frac{n}{n+2}} \equiv L^{\frac{n+2}{2}}-v_{c} T L^{-\frac{n}{2}} .
$$

Taking into account the prefactor in (18), the corresponding set of needed expressions (omitting from now on the subscripts $v_{c} \rightarrow v ; a_{c} \rightarrow a$ for simplicity) can be written using for simplicity the notation

$$
\omega=\frac{X}{\bar{X}} \equiv\left(1-v_{c} T X^{\beta-2}\right)^{-1} \text {. }
$$

One obtains

$$
\begin{aligned}
\partial_{T} U & =U\left(a \beta / 2 \bar{X}^{\beta-1}-\beta / 2 \bar{X}^{\beta / 2-1}\right) v \bar{X}^{\beta-1} \omega^{\beta-1} \\
\partial_{X} U & =U\left(-a \beta / 2 \bar{X}^{\beta-1}+\beta / 2 \bar{X}^{\beta / 2-1}\right)\left(1+v(1-\beta) T \bar{X}^{\beta-2} \omega^{\beta-2}\right) \\
\partial_{X}^{2} U & =U\left(a \beta / 2 \bar{X}^{\beta-1}-\beta / 2 \bar{X}^{\beta / 2-1}\right)^{2}\left(1+v(1-\beta) T \bar{X}^{\beta-2} \omega^{\beta-2}\right)^{2}+ \\
& +U\left(a \beta / 2(1-\beta) \bar{X}^{\beta-2}-\beta / 2(1-\beta / 2) \bar{X}^{\beta / 2-2}\right)\left(1+v(1-\beta) T \bar{X}^{\beta-2} \omega^{\beta-2}\right)^{2}+ \\
& +U\left\{a \beta / 2 \bar{X}^{\beta-1}-\beta / 2 \bar{X}^{\beta / 2-1}\right\}(1-\beta)(2-\beta) v T \bar{X}^{\beta-3} \omega^{\beta-3} .
\end{aligned}
$$

Now, we find an iterative solution of (7) by ordering in decreasing powers of $X$. Assuming before further discussion to consider the kinematical region where

$$
\omega=1+\mathcal{O}\left(T \bar{X}^{\beta-2}\right) \sim 1
$$

and inserting the relations (21) in (7) order by order, and considering the leading terms from all contributions one gets

$$
\begin{aligned}
\frac{1}{U} \partial_{T} U & : v a \beta / 2 \bar{X}^{2 \beta-2}-v \beta / 2 \bar{X}^{3 \beta / 2-2}+\cdots \\
\frac{1-\beta}{U X} \partial_{X} U & :-a \beta / 2(1-\beta) \bar{X}^{\beta-2}+\beta / 2(1-\beta) \bar{X}^{\beta / 2-2}+\cdots \\
\gamma \bar{X}^{\beta-1} \frac{1}{U} \partial_{X} U & :-\gamma\left(a \beta / 2 \bar{X}^{2 \beta-2}-\beta / 2 \bar{X}^{3 \beta / 2-2}\right)+\cdots \\
\frac{1}{U} \partial_{X}^{2} U & : a^{2} \beta^{2} / 4 \bar{X}^{2 \beta-2}-a \beta^{2} / 2 \bar{X}^{3 \beta / 2-1}+\left(a \beta / 2(1-\beta)-\beta^{2} / 4\right) \bar{X}^{\beta-2}-\beta / 2(1-\beta / 2) \bar{X}^{\beta / 2-2}+\cdots \\
\frac{1}{U} \lambda U & : \lambda \bar{X}^{2 \beta-2} .
\end{aligned}
$$

Collecting the leading terms from all contributions to Eq.(7) one gets from the four first orders

$$
\begin{array}{rll}
\bar{X}^{2 \beta-2} & : a^{2} \beta^{2} / 4-v a \beta / 2-\gamma a \beta / 2+\lambda=0 \\
\bar{X}^{\frac{3}{2} \beta-2} & : & v \beta / 2+\gamma \beta / 2-a \beta^{2} / 2=0 \\
\bar{X}^{\beta-2} & : & -a \beta / 2(1-\beta)+a \beta / 2(1-\beta)-\beta^{2} / 4=-\beta^{2} / 4 \\
\bar{X}^{\frac{1}{2} \beta-2} & : & \beta / 2(1-\beta)-\beta / 2(1-\beta / 2)=-\beta^{2} / 4 .
\end{array}
$$

Both first relations are satisfied by the critical condition (17) $v_{c}+\gamma=2 \sqrt{\lambda}=a_{c} \beta$. Hence the consideration of the special solution (18) allowed for one subleading order veryfying the equation. The nonzero remaining orders are $\bar{X}^{\beta-2}, \bar{X}^{\frac{1}{2} \beta-2}$. As we shall see further on, the subsubleading contributions, together with those coming beyond the approximation (22) will be dealt with the linearly $T$-dependent terms. Interestingly enough, these subsubleading powers will be related, in the known cases at least $[3,5]$, to the subleading terms of the universal expansion of the scaling variable. 


\section{EXTENDING GEOMETRIC SCALING}

In order to explore the kinematic range of validity of the scaling variable $\bar{X}(X, T)$ and look for corrections allowing to take into account sub-subleading corrections induced by the set of equations (24), let us recall the previously known results on geometric scaling coming for the studies [3] on nonrunning $(n=0)$ and running $(n=1)$ BK equations and make the appropriate generalization of these known results for generic $n$.

One is led to distinguish 3 regions [5] (we will see that we can add a 4th one) namely, in their order towards the moving tail of the wave, the wave interior region, where geometric scaling has been identified, the leading edge, where diffusive corrections appear, and the very forward region where the nonuniversal initial conditions prevail. Extrapolating for all $n$ the known results for $n=0$ and 1, the wave interior region can be characterized by the kinematic relation $L \sim\left(v_{c} T\right)^{\frac{1}{n+1}}$ and the leading edge region by $L \sim\left(v_{c} T\right)^{\frac{1}{n+1}}+\rho\left(v_{c} T\right)^{\frac{1}{n+2}}$ where $\rho \sim c s t$.

Focussing on the leading edge and extrapolating for all $n$ the Ansatz proposed in refs. $[3,8]$ for the standard running and nonrunning FKPP cases, the generic solution reads

$$
U_{n}(X, T) \propto T^{\frac{1}{n+2}} G_{n}\left(\frac{L-\left(v_{c} T\right)^{\frac{1}{n+1}}+c_{n}(T)}{T^{\frac{1}{n+2}}}\right) e^{\gamma\left\{L-\left(v_{c} T\right)^{\frac{1}{n+1}}+c_{n}(T)\right\}},
$$

where $n=(0,1)$ is the previously defined index for nonrunning and running cases, $\tau_{n}(L, T) \equiv L-\left(v_{c} T\right)^{\frac{1}{n+1}}+c_{n}(T)$ is the original scaling variable extrapolated from the values obtained in [3], $c_{n}(T)$ the corresponding subleading universal contributions to the scaling variable and $\gamma$ a universal critical slope value characterizing the scaling in the wave interior when $z \equiv \tau_{n}(L, T) / T^{\frac{1}{n+2}} \rightarrow 0$. Note the major constraint in this limit $G_{n}(z) \sim z$ which expresses the "absorptive" property [12] due to the nonlinear terms. This is the very same property which is realized in our Ansatz (18), Motivating the absence of a pure exponential term a priori allowed for a solution of a second-order linear equation.

The function $G_{n}$ has been determined for $n=0,1$ (see e.g. [3]), while their precise form in the general case, not relevant for our needs, should not be difficult to be obtained in a similar way.

Within the typical leading edge region where $L \sim\left(v_{c} T\right)^{\frac{1}{n+1}}-c_{n}(T) \tau+\rho_{n} T^{\frac{1}{n+2}}$, where $\rho_{n}$ is a smooth function around some constant value. We see that the expression (25) takes the scaling form

$$
U_{n}(X, T) \propto T^{\frac{1}{n+2}} G_{n}\left(\rho_{n}\right) e^{\gamma \rho_{n} T^{\frac{1}{n+2}}} \sim \frac{1}{\rho_{n}} \tau_{n}(L, T) G_{n}\left(\rho_{n}\right) e^{\gamma \tau_{n}(L, T)} .
$$

Hence in the leading edge region, we can formulate the standard solution with essentially the same original scaling variable.

Strikingly enough, a matching with our obtained solution (18) is obtained in an appropriate kinematic region where

$$
X \sim K v_{c} T ; \quad L \sim\left(K v_{c} T\right)^{\frac{n+2}{2}},
$$

where the constant $K>1$ in the nonrunning case. This kinematic region is always near and forward to the identified leading edge regions characterized (see [3] for $n=0,1)$ by $L \sim v_{c} T^{\frac{1}{n+1}}+\mathcal{O}\left(T^{\frac{1}{n+2}}\right)$. For instance, $L \sim\left(v_{c}+K\right) T$ for the nonrunning case and $L \sim v_{c} T^{2 / 3}$ for the running case. Let us call for convenience this region the extended scaling region [13]. Note also that one identifies

$$
a_{c} \equiv(n+2) \sqrt{\lambda} \sim \gamma \rho_{n} .
$$

Hence requiring a matching between the leading edge and extended scaling regions, and using the scaling variable $\bar{X} \equiv X-v_{c} T X^{\frac{n}{n+2}}$ leads to an extended scaling domain. Interestingly enough the scaling variable gets modified, since

$$
\bar{X} \equiv X-v_{c} T X^{-\frac{1}{n+2}}=L^{\frac{n+2}{2}}-v_{c} T L^{-\frac{n}{2}} .
$$

As an application to the physical cases, in the nonrunning case, one realizes that the scaling variable is the original one, while the overall scaling region has been enhanced thanks to the modification of the scaling function. In the running coupling case, one finds $X-v_{c} T X^{-\frac{1}{3}}=L^{\frac{3}{2}}-v_{c} T L^{-\frac{1}{2}}$, which shows the compatibility with the standard scaling variable $\tau=L-\left(v_{c} T\right)^{1 / 2}$, since $L^{2} \sim v_{c} T$ near the wave front.

We thus obtain a new scaling solution extended in the forward region matching at its lower $X$ boundary with the leading edge. We thus infer that, for all value of $\beta=\frac{2}{2+n}$ the variable $X-v_{c} T X^{1-\beta} \equiv L^{\frac{n}{2}+1}-v_{c} T L^{-\frac{n}{2}}$ is a promising candidate to ensure an extended geometric scaling. 
In order to check the validity of the scaling in this domain, one has to ensure that the approximation (22) is valid for $\bar{X} \propto T$. By inspection of the system of equations (21), one realizes that the correction is of order $\bar{X}^{3 \beta-4}<<$ $\bar{X}^{2 \beta-2} \bar{X}^{\frac{3}{2} \beta-3}$ at large $\bar{X}$, ensuring that the two first relations of (24) are still valid, preserving the enhanced scaling property. Hence beyond the wave interior and the leading edge and before the very forward regions classically analyzed e.g. in Ref.[5], one finds a new extended scaling region for generalized FKPP equations and their traveling wave solutions.

Concerning the subsubdominant orders in (24), they may receive contributions from those $3 \beta-3$ terms coming from (22) (except for the nonrunning case $\beta=1$ ) and those in $\beta-2$ and $\frac{\beta}{2}-2$. A simple way to take these terms into account is to add subleading terms in the $T$-dependence of the scaling variable taken into account by the function $c(T)$ which appears in the leading edge formula (25). For instance, considering

$$
\bar{X}=X-v_{c} T+h \log T+k T^{-\frac{1}{2}}+\cdots \text { for } \beta=1 ; \quad \bar{X}=X-v_{c} T+h T^{1-\beta}+k T^{-\frac{3 \beta}{2}}+\cdots \text { for } \beta<1,
$$

one generates the order $\beta-2$ and $\frac{\beta}{2}-2$. Other inputs will easily ensure the cancellation of the subdominant terms of order $3 \beta-3$ present in the $\beta \neq 1$ cases. Note the interesting fact that the expressions (30) are exactly matching the orders of the well-known subdominant universal terms [3,5]. In the case $\beta \neq 1$, new terms appear which deserve a specific study. We postpone this for further work.

\section{SUMMARY AND OUTLOOK}

Let us summarize our main results:

- Mapping to (s)FKPP universality classes. We introduce a one-parameter indexed by $n$ family of nonlinear equations including and interpolating the Balitsky-Kovchegov equations with nonrunning and running QCD couplings as $\left\{\log Q^{2}\right\}^{-n}$. In the diffusive approximation, this family is exactly mapped to a (s)FKPP equation describing the propagation of radial traveling waves in an absorptive medium of dimensionality $2(n+1) /(n+2)$ and coefficient functions depending on the "space" variable $X=\left\{\log Q^{2}\right\}^{\frac{2}{n+2}}$. The different coefficients verify (except for $n=0$ ) at large $X$ the hierarchy

$$
X^{0}(\text { Diffusion })>>X^{-\frac{n}{n+2}}\left(\text { "Drift") }>>X^{-\frac{2 n}{n+2}}(\text { Birth/Death rate })>>X^{-\frac{3 n}{(n+2)}}\right. \text { (Noise strength) }
$$

- Geometric Scaling Solutions. Using an expansion at large $X$, we find a new geometric-scaling domain forward to the canonical traveling wave front such that $L \sim\left(K v_{c} T\right)^{\frac{2}{n+2}}$, where $T \propto Y$ the rapidity plays the role of "time", $v_{c}$ is the minimal speed (the constant $K$ is arbitrary except that $K>1$ when $n=0$ ) with the scaling solution

$$
U \propto e^{-(n+2) \sqrt{\lambda}\left(L^{\frac{n+2}{2}}-v_{c} T L^{-\frac{n}{2}}\right)^{\frac{1}{n+2}}}\left(L^{\frac{n+2}{2}}-v_{c} T L^{-\frac{n}{2}}\right)^{\frac{1}{n+2}},
$$

where $\lambda$ is a constant depending on the BK equation parameters, see (8).

- Extended Geometric Scaling. Matching with the universal solutions in the near-by less forward leading edge region, the scaling variable $L^{\frac{n+2}{2}}-v_{c} T L^{-\frac{n}{2}}$ is shown to allow for an extension of the geometric-scaling region in a new kinematic domain.

Specifying for the nonrunning case $(n=0)$, one has the same scaling variable as previously, but a new scaling form allowing to extend the geometric scaling region from the wave interior $L \sim v_{c} T$, including the leading edge region with $L \sim\left(v_{c} T\right)+\rho_{0} T^{\frac{1}{2}}$ up to an extended forward region where $L \sim K v_{c} T$ with $K>1$.

For the running and more general $n \neq 0$ cases, one finds starting from the wave interior region $L \sim v_{c} T^{\frac{1}{n+1}}$, scaling in the leading edge region $L \sim\left(v_{c} T\right)^{\frac{1}{n+1}}+\rho_{1} T^{\frac{1}{n+2}}$ up to an extended scaling region where $L \propto\left(v_{c} T\right)^{\frac{2}{n+2}}$.

The output of these properties is interesting in particular for the running coupling case $(n=1)$, which is physically meaningful for the BK equation in QCD. For instance, the parameter $a_{c}=(n+2) \sqrt{\lambda}$ related to an effective slope of the scaling curve is increasing by a factor $\frac{3}{2}$ from nonrunning to the running case, while the leading edge form leads to a constant slope. It would be worth comparing this prediction with a simulation of the BK equation [14] where a similar effect has been noticed. 
On the phenomenological ground, which is not our subject in the present paper, there are quite a few issues which deserve to be studied. Last but not least, it would be worthwhile to confront the new extended scaling properties, in particular the new scaling variable $\bar{X}=X-v_{c} T X^{-\frac{1}{3}} \equiv L^{\frac{3}{2}}-\left(v_{c} T\right) L^{-\frac{1}{2}}$, with data using e.g. the method of Refs.[15]. Also, geometric scaling in other reactions, such as hard diffractive events [16], would be welcome.

On the theoretical ground, some studies remain to be done in the new framework. For instance, the effect of a cut-off [8] could be analyzed using the general solution (16) with probably complex conjugate functions and match with the cut-off region. Also, the investigation of subleading universal terms is possible, thanks to appropriate modification of the time dependence of the scaling variable. It could be worthwhile to merge the new scaling approach with the studies on the parametric form of the traveling waves [17].

One would also like to go beyond the diffusive aproximation. At the present stage of our theoretical knowledge, the BK equation has been corrected for the full nest-leading log contributions [18]. Indeed, the matching with the standard universal formulation ensures that one should not expect dramatic effects, except for the neat difference between the fixed and running coupling cases [19], but this dedicated study deserves to be done in the future. In any case, it would be interesting to see whether and how it works.

\section{Aknowledgements}

The autor would like to thank Guillaume Beuf, Andrzej Bialas and Cyrille Marquet for useful discussions and (for C.M.) a careful critical reading of the manuscript. During the completion of this work, he was partly supported by the "Partenariat Hubert Curien - Polonium 2009" by the Ministry of Foreign and European Affairs, France, and thanks the Institute of physics of the Jagiellonian university in Cracow for hospitality.

* Electronic address: robi.peschanski@cea.fr

[1] A. M. Staśto, K. Golec-Biernat, and J. Kwiecinski, Phys. Rev. Lett. 86, 596 (2001) [arXiv:hep-ph/0007192].

[2] I. Balitsky, Nucl. Phys. B463 (1996) 99; Y. V. Kovchegov, Phys. Rev. D60 (1999) 034008; Phys. Rev. D61 (2000) 074018.

[3] S. Munier and R. Peschanski, Phys. Rev. Lett. 91, 232001 (2003) [arXiv:hep-ph/0309177]; Phys. Rev. D69, 034008 (2004) [arXiv:hep-ph/0310357]; Phys. Rev. D70, 077503 (2004) [arXiv:hep-ph/0310357].

[4] R. A. Fisher, Ann. Eugenics 7 (1937) 355; A. Kolmogorov, I. Petrovsky, and N. Piscounov, Moscou Univ. Bull. Math. A1 (1937) 1; M. Bramson, Mem.Am.Math.Soc. 44, 285 (1983).

[5] For a comprehensive review: Ute Ebert, Wim van Saarloos, Physica D 146, 1-99 (2000) convergence towards uniformly translating pulled fronts arXiv:cond-mat/0003181.

[6] L. N. Lipatov, Sov. J. Nucl. Phys. 23, (1976) 338; E. A. Kuraev, L. N. Lipatov and V. S. Fadin, Sov. Phys. JETP 45, (1977) 199; I. I. Balitsky and L. N. Lipatov, Sov. J. Nucl. Phys. 28, (1978) 822.

[7] G. Beuf, "An alternative scaling solution for high-energy QCD saturation with running coupling," arXiv:0803.2167 [hep-ph].

[8] E. Brunet and B. Derrida, Phys. Rev. E57 (1997) 2597 [arXiv:cond-mat/0005362]; Comp. Phys. Comm. 121 (1999) 376 [arXix:cond-mat/0005364].

A. H. Mueller and A. I. Shoshi, Nucl. Phys. B 692 (2004) 175 [arXiv:hep-ph/0402193];

[9] E. Iancu, A. H. Mueller and S. Munier, Phys. Lett. B 606 (2005) 342 [arXiv:hep-ph/0410018]. S. Munier, Nucl. Phys. A 755 (2005) 622 [arXiv:hep-ph/0501149].

[10] A. Dumitru, E. Iancu, L. Portugal, G. Soyez and D. N. Triantafyllopoulos, JHEP 0708, 062 (2007).

[11] G. Beuf, Nucl. Phys. A 810, 142 (2008).

[12] A.H. Mueller, D.N. Triantafyllopoulos, Nucl. Phys. B 640 (2002).

[13] The term "extended" geometrical scaling has been already used in

E. Iancu, K. Itakura and L. McLerran, Nucl. Phys. A 708, 327 (2002).

The authors have shown that for $1 \leq \log \left(Q^{2} / Q_{s}^{2}\right) \leq \log \left(Q_{s}^{2}\right) / \Lambda$, where $Q_{s}(Y)$ is the saturation scale, the solution to the linear BFKL equation shows approximate geometric scaling.

[14] J. L. Albacete and Y. V. Kovchegov, Phys. Rev. D 75, 125021 (2007).

[15] F. Gelis, R. B. Peschanski, G. Soyez and L. Schoeffel, Phys. Lett. B 647, 376 (2007); G. Beuf, R. Peschanski, C. Royon and D. Salek, Phys. Rev. D 78, 074004 (2008).

[16] C. Marquet and L. Schoeffel, Phys. Lett. B 639, 471 (2006).

[17] R. B. Peschanski, Phys. Lett. B 622, 178 (2005); C. Marquet, R. B. Peschanski and G. Soyez, Phys. Lett. B 628, 239 (2005).

[18] I. Balitsky, Phys. Rev. D 75, 014001 (2007);

Y. V. Kovchegov and H. Weigert, Nucl. Phys. A 784, 188 (2007);

I. Balitsky and G. A. Chirilli, Phys. Rev. D 77, 014019 (2008).

[19] G. Beuf, R. B. Peschanski and S. Sapeta, Phys. Rev. D 75, 114001 (2007). 